Review began 12/27/2021 Review ended 01/07/2022 Published 01/10/2022

๑) Copyright 2022

Subramanian et al. This is an open acces article distributed under the terms of the Creative Commons Attribution License CCBY 4.0., which permits unrestricted use, distribution, and reproduction in any medium, provided the original author and source are credited.

\section{Prevalence of Anemia Among Adolescent Girls Residing in Rural Haryana: A Community-Based Cross-Sectional Study}

Muthathal Subramanian $^{1}$, Sumit Malhotra ${ }^{2}$, Shashi Kant ${ }^{2}$, Kiran Goswami $^{2}$, Vanamail Perumal ${ }^{3}$, Gaurishanker Kaloiya ${ }^{4}$

1. Community Medicine, Panimalar Medical College Hospital and Research Institute, Chennai, IND 2. Centre for Community Medicine, All India Institute of Medical Sciences, New Delhi, IND 3. Statistics \& Demography, Obstetrics \& Gynaecology, All India Institute of Medical Sciences, New Delhi, IND 4. Clinical Psychology, All India Institute of Medical Sciences, New Delhi, IND

Corresponding author: Sumit Malhotra,drsumitaiims@gmail.com

\section{Abstract}

\section{Background}

Anemia continues to be a major public health problem in India despite multiple initiatives to address it among various vulnerable groups including adolescents.

\section{Aim}

This study was conducted to assess the prevalence of anemia among rural adolescent girls who had attained menarche.

\section{Methods}

The community-based cross-sectional study was conducted in 28 villages of Ballabgarh Block of district Faridabad, Haryana. From the computerized Health Management Information System data (HMIS), a random list of 363 adolescent girls was generated. Adolescent girls who had attained menarche were included in the study. Hemoglobin level was measured for all the consented or assented participants using a digital hemoglobinometer (HemoCue201+ photometer, HemoCue AB, Angelholm, Sweden).

\section{Results}

A total of 272 participants were enrolled in the study. Mean (SD) age at menarche was 13.2 (1.2) years. Prevalence of anemia among adolescent girls who had attained menarche was observed to be $71.7 \%$ ( $95 \%$ CI: 66.3 - 77.1) as per the WHO classification. Among the 195 anemic adolescent girls, severe, moderate, and mild anemia was observed in $4.8 \%, 41.2 \%$, and $25.7 \%$, respectively. In multivariable analysis, after adjusting for the age, the mother's education was significantly associated with anemia (Adjusted Odds Ratio $=0.46$, 95\% CI: $0.22-0.96$, p-value $=0.04)$.

\section{Conclusion}

The prevalence of anemia among rural adolescent girls who had attained menarche was high. Mother's education status had a protective effect on anemia among adolescent girls.

Categories: Preventive Medicine, Epidemiology/Public Health, Hematology

Keywords: prevalence, rural, menarche, adolescent girls, anemia

\section{Introduction}

There are 253 million adolescents in India, and 72\% of them reside in rural areas [1]. Anemia is a serious public health challenge in India, even among adolescents [2]. According to National Family Health Survey-4 (2015-2016), the prevalence of anemia, i.e. hemoglobin level < 12gm/dL among adolescent girls (15-19 years), was $54 \%$ [3].

The adolescence period especially in girls is vulnerable to anemia due to the increased demand during the period of a growth spurt, inadequate dietary intake, vulnerability for helminthic infestation, and increased loss of iron during menstruation. Adolescent girls have a median iron loss of 12.5-15 mg per month during menstruation [4].

The nutritional concerns of adolescent girls, including anemia, are addressed by several flagship schemes and programs of the Government of India. These include the Integrated Child Development Services (ICDS) scheme, the Rajiv Gandhi Scheme for Empowerment of Adolescent Girls (RGSEAG)-SABLA, and more 
recently, POSHAN Abhiyaan and Anemia Mukt Bharat [5-7]. Since there are multiple programs addressing anemia, many specifically focusing on adolescent girls, there is a need for monitoring the prevalence of anemia for assessing the impact of such measures. Adolescent girls living in rural areas, especially those who have attained menarche, constitute the most vulnerable subgroup.

The Comprehensive National Nutrition Survey (CNNS, 2017) in the state of Haryana estimated anemia using venous samples in adolescent girls as $41 \%$, almost twice than prevalence seen in boys (22\%) [8]. Anemia causes easy fatigability, menstrual irregularities, and poor physical as well as mental health outcomes. Among adolescents in addition to this, there is a high chance of poor literacy outcomes. There is a paucity of literature about anemia among girls who had attained menarche. Against this background, this study aimed to estimate the prevalence of anemia and factors associated with it among adolescent girls (10-19 years) who had attained menarche and lived in rural areas of Ballabgarh Block of district Faridabad, Haryana.

\section{Materials And Methods}

A community-based cross-sectional study was conducted in 28 villages of Ballabgarh Block of district Faridabad, Haryana from November to December 2017. The total population of these villages in (2016-17) was 99,747. Data were obtained from the well-established database, i.e., Health Management Information System (HMIS). A computerized HMIS contained health and demographic data of all residents of these 28 villages. A more detailed description of the study site is available elsewhere [9]. The inclusion criterion was adolescent girls (10-19 years), who had attained menarche. Adolescents that were pregnant, unable to comprehend, or not contactable after three home visits were excluded. Assuming the prevalence of anemia (p) among adolescent girls as $87 \%$ [10], relative precision of 5\%, 95\% confidence level, and $15 \%$ nonresponse rate, the minimum required sample size was 242 . The menarcheal status among adolescents was unknown. Hence, the sample size was further inflated by $50 \%$. The final required sample size thus obtained was 363 . There were 7,748 adolescent girls in the HMIS database. A list of total adolescent girls $(\mathrm{N}=7,748)$ was generated from the HMIS database of the rural field practice area, Ballabgarh Block. Using this sampling frame, a simple random sample of 363 adolescent girls was drawn in the Microsoft (MS) Excel 2016 (Microsoft ${ }^{\circledR}$ Corp., Redmond, WA) using =RAND () function, meaning random. This function was used to generate a random list of adolescent girls from the sampling frame. Once the random list was made, girls were sorted village-wise and were approached within the community with the help of a local village-level health worker for the interview.

\section{Data collection}

The study was conducted for two months by the trained investigator [MS]. A self-developed, pre-tested, semi-structured questionnaire that contained items relevant to study objectives viz. demographic details (including socioeconomic status), menstrual history and intake of prophylactic iron and folic acid supplements, and deworming was used. The study instrument was not validated separately, though was pretested and piloted in the adjacent study area before use. Hemoglobin level was measured by a digital hemoglobinometer by the study investigator [MS]. Training and supervisory support for the execution of the data collection work was provided by senior investigators within the team [SM, SK, KG].

Hemoglobin estimation (gm/dl) was done by using the digital hemoglobinometer method, i.e., Hemocue201+ (Hemocue AB, Angelhom, Sweden). This is a point of care diagnostic method utilized in field settings that are operated through the battery. It works on the principle of modified azide methemoglobin determination using a specially designed micro cuvette with dried reagents. The hemoglobin method is estimated using absorbance levels at two wavelengths of $570 \mathrm{~nm}$ and $880 \mathrm{~nm}$ for turbidity compensation in the blood sample [11]. Using a sterilized gauge piece and lancet, the initial two drops of blood were discarded, and the third drop of blood was placed in the HemoCue slide by the investigator (MS), and then in the digital hemoglobinometer. It displayed hemoglobin levels immediately which was noted in the log sheet of the study participant and informed to the participant.

Anemic adolescents were prescribed tablet IFA (Iron Folic Acid). Those requiring further management were referred to the nearest government health facility. The socio-economic status was assessed using the Uday Pareek Scale updated for the year 2017 [12]. Participants in the random list were paid a home visit and screened for eligibility criteria. The participants and available parents/guardians were explained about the study objective and procedure. The assent was taken from the participants if they were younger than 18 years. Written informed consent was taken from the study participants aged 18 years or older and the participant's parents/guardians if the age of the participant was less than 18 years.

The severity of anemia was classified as per the World Health Organization (WHO) recommendation. In brief, anemia was classified as mild if the hemoglobin level was 11.0-11.4 gm/dL for those aged 10 to 11 years, and 11.0-11.9 gm/dL for those aged 12-19 years. Anemia was classified as moderate when the hemoglobin level was 8.0-10.9 gm/dL, and severe if the hemoglobin level was $<8 \mathrm{gm} / \mathrm{dL}$ [2].

Institute Ethics Committee (IEC) approved the study protocol (Reference number: IECPG-224/23.08.2017, RT-07/07.09.2017) 


\section{Cureus}

\section{Statistical methods}

Data were entered in Microsoft Excel 2010 and cleaned data were analyzed using STATA version 12.0 statistical software (StataCorp LLC, College Station, TX). Continuous variables were tested for normality assumption using the Kolmogorov-Smirnov test. For normally distributed data, descriptive measures such as mean, standard deviation (SD), and range values were calculated. For non-normally distributed data, median and inter-quartile range (IQR) were calculated. Median values were compared using the Kruskal-Wallis test or Wilcoxon signed rank-sum test as appropriate. For categorical data, associations were ascertained using Chi-square/Fisher's Exact test as appropriate and odds ratio with 95\% confidence interval (CI) limits were calculated. Bivariable analysis was performed to find an association between anemia and a few selected variables like age, education, mother's education, and socioeconomic status. Significant variables (p-value $\leqslant$ 0.2 ) were entered in the multivariable logistic regression model to calculate the adjusted odds ratio and $95 \%$ CI. A p-value $<0.05$ was considered statistically significant.

\section{Results}

There were 274 eligible participants out of a total of 363 participants. Two eligible participants refused to give consent. Finally, 272 participants were included in the study. The majority of them (67\%) were in the late adolescence group (15-19 years). The mean (SD) age of the participants was $15.5( \pm 1.8)$ years. The majority $(88.2 \%)$ of the participants were students, and $44.9 \%$ were attending middle school. A total of $2.2 \%$ of adolescent girls were married. A total of $43 \%$ of mothers of the participants were illiterate, $21.7 \%$ had completed primary schooling, and $2.6 \%$ of them were graduates. According to the Udai Pareek Scale [12], the majority (48.2\%) of the participants belonged to the middle class and $38.2 \%$ to the lower middle class. Table 1 shows the selected demographic details of the participants. 


\section{Cureus}

\begin{tabular}{|c|c|c|}
\hline Variable & Category & Number (\%) \\
\hline \multirow{2}{*}{ Age group } & Early adolescence (10-14 years) & $90(33.0)$ \\
\hline & Late adolescence (15-19 years) & $182(67.0)$ \\
\hline \multirow{5}{*}{ Education (completed school years) } & Illiterate & $1(0.0)$ \\
\hline & Primary & $6(2.0)$ \\
\hline & Middle school & $122(45.0)$ \\
\hline & High school & $89(33.0)$ \\
\hline & Secondary school & $54(20.0)$ \\
\hline \multirow{2}{*}{ Occupation of the participant } & Student & $240(88.0)$ \\
\hline & Others* & $32(12.0)$ \\
\hline \multirow{2}{*}{ Marital status } & Unmarried & $266(98.0)$ \\
\hline & Married & $6(2.0)$ \\
\hline \multirow{3}{*}{ Religion } & Hindu & $266(98.0)$ \\
\hline & Muslim & $4(1.0)$ \\
\hline & Sikh & $2(1.0)$ \\
\hline \multirow{6}{*}{ Education of the participant's mother } & Illiterate & $117(43.0)$ \\
\hline & Primary & $58(21.0)$ \\
\hline & Middle school & $40(15.0)$ \\
\hline & High school & $29(11.0)$ \\
\hline & Secondary school & $21(8.0)$ \\
\hline & Graduate & $7(3.0)$ \\
\hline \multirow{4}{*}{ Socio-economic status according to Udai Pareek Scale } & Upper middle (33-42) & $34(13.0)$ \\
\hline & Middle (24-32) & $131(48.0)$ \\
\hline & Lower middle (13-23) & $104(38.0)$ \\
\hline & Lower (<13) & $3(1.0)$ \\
\hline
\end{tabular}

TABLE 1: Distribution of the study participants by socio-demographic characteristics $(N=272)$

The mean (SD) hemoglobin level of the participants was $10.9( \pm 1.64) \mathrm{gm} / \mathrm{dL}$. The prevalence of anemia among adolescent girls who had attained menarche was 71.7\% (95\% CI: $66.3-77.1$ ) as per the WHO classification. Among the 195 anemic participants, 13 (6.7\%), 112 (57.4\%) and 70 (35.9\%) had severe, moderate and mild anemia, respectively. The difference between the severity of anemia and age groups was statistically not significant $(\mathrm{p}$-value $=0.9)($ Table 2$)$. 


\section{Cureus}

\begin{tabular}{|c|c|c|c|c|c|}
\hline Age group in years* & Normal & Mild & Moderate & Severe & Total \\
\hline $10-14$ n (\%) & $27(30.0)$ & 24 (26.7) & 35 (38.9) & $4(4.4)$ & $90(100)$ \\
\hline$\geq 15 \mathrm{n}(\%)$ & $50(27.5)$ & $46(25.3)$ & $77(42.3)$ & $9(5.0)$ & $182(100)$ \\
\hline n (\%) (95\% Confidence & $77(28.3)(22.7-33.3)$ & $70(25.7)(19.9-30$. & $112(41.2)$ & $13(4.8)(2.3$ & $272(10$ \\
\hline
\end{tabular}

TABLE 2: Distribution of category of anemia by age group $(N=272)$

${ }^{*} p$-value $=0.9$ using Chi-square test, Chi-square value -0.38 , degree of freedom $(\mathrm{df})=3$

Among the school-going participants, 78 of them (29.9\%) received and consumed iron-folic acid (IFA) supplements. The reason for not consuming was side effects (6.1\%), and for the rest of the participants studied in the private schools. Deworming was done periodically among $26.4 \%$ of participants. Self-reported symptoms such as premenstrual syndrome (61\%), and health problems during menstruation (73\%) were found among the study participants. Less than $1 / 6$ th (13.3\%) of the participants had an irregular menstrual history in terms of regularity of the menstrual cycle and menstrual blood flow.

The prevalence of anemia was more than $50 \%$ among late adolescents. The prevalence of anemia was not associated with age category. The participants who were educated above middle school, belonged to lower socio-economic status, and whose mothers were illiterate had a higher prevalence of anemia in each anemia category. However, the difference was statistically not significant (Table 3).

\begin{tabular}{|c|c|c|c|c|c|}
\hline Variable & Category (n) & Anemia present $(n=195) n(\%)$ & Unadjusted odds ratio & $95 \% \mathrm{Cl}$ & $\mathrm{p}$-value \\
\hline \multirow{2}{*}{ Age } & Early adolescence (90) & $63(32.0)$ & 1.00 & - & - \\
\hline & Late adolescence (182) & $132(68.0)$ & 1.13 & $0.64-1.97$ & 0.66 \\
\hline \multirow{2}{*}{ Education of the participant } & $\leq 8^{\text {th }}$ Class (129) & $89(46.0)$ & 1.00 & - & - \\
\hline & $\geq 9^{\text {th }}$ class $(143)$ & $106(54.0)$ & 1.28 & $0.75-2.18$ & 0.34 \\
\hline \multirow{3}{*}{ Education of the mother } & Illiterate (117) & $90(46.0)$ & 1.00 & - & - \\
\hline & $1-8^{\text {th }}$ class $(98)$ & $69(35.0)$ & 0.82 & $0.44-1.51$ & 0.53 \\
\hline & $9^{\text {th }}$ and above (57) & $36(18.0)$ & 0.55 & $0.27-1.09$ & $0.08^{*}$ \\
\hline \multirow{2}{*}{ Socio-economic status } & Middle and upper middle (165) & $114(58.0)$ & 1.00 & - & - \\
\hline & Lower and lower middle (107) & $81(42.0)$ & 1.39 & $0.80-2.41$ & 0.23 \\
\hline
\end{tabular}

\section{TABLE 3: Distribution of anemia by selected socio-demographic variables}

* $p$-value $\leq 0.20$, unadjusted odds ratio for reference category used for comparison is marked as 1.00

Bivariable analysis between anemia and selected socio-demographic variables revealed that the education of the participant's mother was associated with anemia ( $\mathrm{p}$-value $\leqslant 0.20)$ (Table 3). Logistic regression analysis between the education of the participant's mother and anemia after adjusting for the participant's age was performed. The odds of having anemia among the participants born to educated mothers was $54 \%$ less compared to participants born to illiterate mothers (Table 4). 


\section{Cureus}

\begin{tabular}{|c|c|c|c|c|c|}
\hline Variable & Category (n) & Anemia present $(n=195) n(\%)$ & Adjusted odds ratio & $95 \% \mathrm{Cl}$ & $p$-value \\
\hline \multirow{3}{*}{ Education of the mother } & Illiterate (117) & $90(46.0)$ & 1.00 & - & - \\
\hline & $1-8^{\text {th }}$ class $(98)$ & $69(35.0)$ & 0.78 & $0.42-1.46$ & 0.44 \\
\hline & $9^{\text {th }}$ and above (57) & $36(18.0)$ & 0.46 & $0.22-0.96$ & $0.04^{*}$ \\
\hline
\end{tabular}

\section{TABLE 4: Logistic regression between anemia and education of the mother}

${ }^{*} \mathrm{p}$-value $<0.05$ after adjusting for participant's age in completed years, adjusted odds ratio for reference category used for comparison is marked as 1.00

There was no significant association between IFA intakes through Weekly Iron and Folic Acid Supplementation (WIFS) in school, deworming status, age at menarche, menstrual history, menstrual disorders viz. irregular cycles, hypomenorrhea and anemia.

\section{Discussion}

This study was conducted to assess the prevalence of anemia among adolescent girls who had attained menarche and were living in a rural area of Haryana. The hemoglobin levels as prescribed by the WHO were used to diagnose anemia using a digital hemoglobinometer in this study. For girls aged 10-11 years, hemoglobin level $<11.5 \mathrm{gm} / \mathrm{dL}$, and for girls aged 12 years and above, hemoglobin level $<12.0 \mathrm{gm} / \mathrm{dL}$ was used for diagnosing anemia [2]. The prevalence of anemia was 71.7\% (95\% CI: 66.3 - 77.1). A communitybased study in 2017 conducted among rural adolescent girls (12-19 years) reported a high prevalence of anemia (88.3\%). The study population in this study was school girls from Haryana [13]. A higher prevalence similar to this study was reported from other states also such as by Ahankari et al. [10] in Maharashtra (87\%), Rati and Jawadagi [14] in Karnataka (80\%), Koushik et al. [15] in Andhra Pradesh (77.3\%), and Gupta et al. [16] in Chhattisgarh (76.3\%). The higher prevalence of anemia in the present study could be due to the inclusion of late adolescents as the study population. Late adolescents experience physical and biological changes following puberty that increase the demand for nutrients. Failure to provide the increased demand for iron could lead to anemia among late adolescents.

The reported range of anemia among adolescent girls varies with the study settings, i.e., community-based, school-based, or hospital-based. Among community-based rural Indian studies, the reported prevalence of anemia varies from $21 \%$ to $90 \%$ [10, 17-19]. The reported prevalence of anemia is still higher (44\% to $100 \%$ ) among school-based studies in India $[13,14,16,20,21]$. Two studies from the health care facility reported the prevalence of $48.6 \%$ [22] and 77\% [15] among adolescent girls from southern India. Thus, irrespective of the study setting, the prevalence of anemia among adolescent girls in India is unacceptably high.

A school-based study that had used the same tool as in the current study for estimation of hemoglobin level, reported the prevalence of anemia as $44 \%$. The lower prevalence observed in that study could be due to the exclusion of adolescents aged 15 years or older [20].

The prevalence of severe anemia in the present study was $4.8 \%$. This study's findings were unlike other studies that have reported a much higher figure $(7.1 \%$ to $13 \%)[13-15,18,21,22]$. The precise reason for such a difference is not obvious. However, part of the differences could be explained by the differences in the study setting, and tool used to measure hemoglobin levels. Hemocue201+ has high sensitivity (93\%), acceptable specificity $(76 \%)$, and moderate concordance with the gold standard Sysmex autoanalyzer for measuring hemoglobin [23].

The mother's educational status had a protective effect on anemia in an adolescent girl. Other studies have also reported similar findings [16, 24]. The beneficial effect of a mother's education has been reported on many other health parameters, including infant and child mortality. A mother's education may be reflective of an overall higher level of socio-economic status that in itself has a protective effect against anemia. The impact of socio-economic status on anemia among adolescent girls is well established [14, 16, 21, 22, 24].

There was no association between anemia and iron-folic acid intake, deworming status, menarcheal age, menstrual history, and menstrual disorders. This finding was contrary to the findings reported by other studies. Studies have reported that menstrual factors such as usage of more pads per day ( $>2$ pads), increased flow per cycle ( $\geqslant 5$ days) had a significant association with anemia among adolescent girls [14, 20, 24].

\section{Limitations}

The findings of this study may not be extrapolatable to urban areas and also, they can be generalized only to the adolescent girls who attained menarche within rural communities of Haryana. The self-reported history 


\section{Cureus}

of deworming and iron-folic acid intake, and menstrual problems were taken, which had the possibility of recall bias. We did not ascertain the etiology of anemia, i.e., microscopic typing, and serum indicators that may be needed for determining the burden of different types of anemia in this area. The sample size was calculated for the primary objective that was related to the prevalence of anemia, hence it might be inadequate to establish the association between selected variables and anemia. Future comprehensive studies will be needed to gauze the magnitude from multiple settings and geographic regions along with biochemical analysis to present the burden of specific types of anemia. Our study had a few strengths also. Simple random selection from a known sampling frame and negligible refusal rate ensured that the findings were extrapolatable to the area from which the samples were drawn. A digital hemoglobinometer was used whose sensitivity and specificity in field conditions are acceptable. Hence, the prevalence rate seems to be valid.

\section{Conclusions}

The prevalence of anemia among rural adolescent girls who had attained menarche was high. Mother's education status had a protective effect on anemia among adolescent girls. Involvement of mothers in educating about corrective measures for anemia reduction should be promoted. The etiology of anemia among these rural adolescent girls who had attained menarche needs to be investigated and appropriate preventive and therapeutic measures may be instituted.

\section{Appendices}




\section{Cureus}

\section{PARTICIPANT DEMOGRAPHIC DETAILS:}

1 Age in completed years: If available, Date of birth: 2

Participant ID/ Village name/ Household no:

3 Marital status: [0-unmarried/1-married]

Education of the participant: (completed schooling) [0- illiterate/1- $1^{\text {st }}$

4 to $5^{\text {th }} / 2-6^{\text {th }}$ to $8^{\text {th }} / 3-9^{\text {th }}$ to $10^{\text {th }} / 4-11^{\text {th }}$ to $12^{\text {th }} / 5$ - graduate]

Occupation of the participant: [0- student/1- housemaker/2- labor/3 others (specify)]

Education of the mother/ guardian of the participant: [0-illiterate/1-1 ${ }^{\text {st }}$

6 to $5^{\text {th }} / 2-6^{\text {th }}$ to $8^{\text {th }} / 3-9^{\text {th }}$ to $10^{\text {th }} / 4-11^{\text {th }}$ to $12^{\text {th }} / 5$-graduate]

7 Religion: [0-Hindu/1-Christian/2-Muslim/3-sikh /4-others(specify)]

\section{DETAILS OF MENSTRUAL HISTORY:}

16

17

19

20

\section{MEDICATION HISTORY RELATED TO WIFS:}

21

$22 b$
10

Land: [0- no land/1- $<1$ acre/2- 1-5 acre/3- 5-10 acre/4- 10-15 acre/5$15-20$ acre/6- $20 \&>$ acre]

Social participation: [0- none/1- member of 1 organization /2- member

11 of $>1$ organization / 3- office holder in such an organization/4- wide public leader] House: [0- no house/1- hut/2- kutcha/3- mixed/ 4- pukka/5- mansion]

13

Farm power:[1- no draught animals/2- 1-2 draught animals/4- 3-4 draught animals/6- 5-6 draught animals]

Material possessions: [0-bullock cart/1-cycle/2-radio/3-chairs/4-mobile phone/5-television/ 6-refrigerators]
Dysmenorrhea /Menorrhagia / Hypo menorrhea/Oligomenorrhea/ Polymenorrhea/ Premenstrual symptoms/ Backache /Others (specify)
Have you attained menarche? [1yes.0-no] If yes, go to the next question.

Age at menarche in completed years

What is your average duration of menstruation in days per menstrual cycle?

In the last period, how many days was the menstrual flow?

What is the interval in days between two cycles?

What are the health-related problems you faced during menstruation?
Family type: [1- single/2- joint or size up to 5 or any other distinctive atures/3- joint]

Caste: [1- SC/2- low caste/3- artisan caste/ 4- agriculture caste/5-

History of deworming in the last 6

months [1-yes.0-no]

Have you taken iron-folic acid (IFA)

through WIFS in school? [1-yes.0-no]

If yes, the number of tablets consumed

in the last 3 months

Hemoglobin value in $\mathrm{gm} / \mathrm{dL}$

\section{TABLE 5: Study Questionnaire/ Interview Proforma}

\section{Additional Information \\ Disclosures}

Human subjects: Consent was obtained or waived by all participants in this study. Institute Ethics Committee issued approval IECPG-224/23.08.2017, RT-07/07.09.2017. All India Institute of Medical Sciences, 
Institute Ethics Committee approved the study protocol. Informed Consent and Assent were obtained as appropriate from the parents or guardians and participants. Animal subjects: All authors have confirmed that this study did not involve animal subjects or tissue. Conflicts of interest: In compliance with the ICMJE uniform disclosure form, all authors declare the following: Payment/services info: All authors have declared that no financial support was received from any organization for the submitted work. Financial relationships: All authors have declared that they have no financial relationships at present or within the previous three years with any organizations that might have an interest in the submitted work. Other relationships: All authors have declared that there are no other relationships or activities that could appear to have influenced the submitted work.

\section{References}

1. Office of the Registrar General \& Census Commissioner, India. Population enumeration data (final population). (2011). Accessed: March 18, 2021:

http://www.censusindia.gov.in/2011census/population_enumeration.html.

2. World Health Organization: Haemoglobin Concentrations for the Diagnosis of Anemia and Assessment of Severity. World Health Organization, 2011.

3. International Institute for Population Sciences (IIPS) and ICF. National family health survey (NFHS-4) 201516: India. (2017). Accessed: March 18, 2021: https://dhsprogram.com/pubs/pdf/FR339/FR339.pdf.

4. DeMaeyer EM, Dallman P, Gurney JM, Hallberg L, Sood SK, Srikantia SG: Preventing and Controlling Iron Deficiency Anemia Through Primary Health Care. A Guide for Health Administrators and Programme Managers. World Health Organization, 1989.

5. Jan Andolan Guidelines. POSHAN Abhiyaan - Ministry of Women and Child Development, Government of India. (2021). Accessed: February 16, 2021: https://icds-wcd.nic.in/nnm/home.htm.

6. Anemia Mukt Bharat. Intensified National Iron Plus Initiative (I-NIPI) for programme managers . (2018). https://anemiamuktbharat.info/wp-content/uploads/2019/09/Anemia-Mukt-Bharat-Brochure_English.pdf.

7. Technical handbook on anemia in adolescents. Weekly iron and folic acid supplementation programme . (2021). Accessed: January 10, 2022:

https://www.nhm.gov.in/images/pdf/programmes/wifs/guidelines/technical_handbook_on_anaemia.pdf.

8. Ministry of Health and Family Welfare (MoHFW), Government of India. Comprehensive National Nutrition Survey (CNNS) - 2016-2018. (2021). Accessed: December 29, 2021: https://nhm.gov.in/WriteReadData/1892s/1405796031571201348.pdf.

9. Kant S, Misra P, Gupta S, et al.: The Ballabgarh Health and Demographic Surveillance System (CRHSPAIIMS). Int J Epidemiol. 2013, 42:758-768. 10.1093/ije/dyt055

10. Ahankari AS, Myles PR, Fogarty AW, Dixit JV, Tata LJ: Prevalence of iron-deficiency anaemia and risk factors in 1010 adolescent girls from rural Maharashtra, India: a cross-sectional survey. Public Health. 2017, 142:159-166. 10.1016/j.puhe.2016.07.010

11. Hemoglobin test - HemoCue Hb 201+ System. (2021). Accessed: January 10, 2022: https://www.hemocue.com/en/solutions/hematology/hemocue-hb-201plus-system.

12. Singh T, Sharma S, Nagesh S: Socio-economic status scales updated for 2017. Int J Res Med Sci. 2017, 5:3264-3267.

13. Gupta A, Kapil U, Lal PR: Prevalence of anemia amongst school going adolescent girls residing in rural Block of Haryana, India. Curr Dev Nutr. 2020, 4:198. 10.1093/cdn/nzaa043_049

14. Rati SA, Jawadagi S: Prevalence of anemia among adolescent girls studying in selected schools . Int J Sci Res. 2012, 3:1237-1242.

15. Koushik NK, Bollu M, Ramarao NV, Nirojini PS, Nadendla RR: Prevalence of anemia among the adolescent girls: a three months cross-sectional study. World J Pharm Pharm Sci. 2014, 3:827-836.

16. Gupta V, Chavhan PS, Singh SP, Umate L: Prevalence of anemia among adolescent girls in rural population of Rajnandgaon, Chhattisgarh, India. J Med Sci Clin Res. 2017, 5:17369-17374. 10.18535/jmscr/v5i2.26

17. Biradar SS, Biradar SP, Alatagi AC, Wantamutte AS, Malur PR: Prevalence of anaemia among adolescent girls: a one year cross-sectional study. J Clin Diagn Res. 2012, 6:372-377.

18. Toteja GS, Singh P, Dhillon BS, et al.: Prevalence of anemia among pregnant women and adolescent girls in 16 districts of India. Food Nutr Bull. 2006, 27:311-315. 10.1177/156482650602700405

19. Siva PM, Sobha A, Manjula VD: Prevalence of anaemia and its associated risk factors among adolescent girls of central Kerala. J Clin Diagn Res. 2016, 10:19-23. 10.7860/JCDR/2016/20939.8938

20. Rakesh PS, George LS, Joy TM, George S, Renjini BA, Beena KV: Anemia among school children in Ernakulam district, Kerala, India. Indian J Hematol Blood Transfus. 2019, 35:114-118. 10.1007/s12288-0181001-6

21. Dhillon PK, Kumar B, Verma HK: Prevalence of anemia in view of socio-demographic and health status of adolescent girls enrolled in government school at border-belt of Indian Punjab. Ecol Food Nutr. 2021, 60:198-211. 10.1080/03670244.2020.1824160

22. Chandrakumari AS, Sinha P, Singaravelu S, Jaikumar S: Prevalence of anemia among adolescent girls in a rural area of Tamil Nadu, India. J Family Med Prim Care. 2019, 8:1414-1417. 10.4103/jfmpc.jfmpc_140_19

23. Yadav K, Kant S, Ramaswamy G, et al.: Validation of point of care hemoglobin estimation among pregnant women using digital hemoglobinometers (HemoCue 301 and HemoCue 201+) as compared with autoanalyzer. Indian J Hematol Blood Transfus. 2020, 36:342-348.

24. Pattnaik S, Patnaik L, Kumar A, Sahu T: Prevalence of anemia among adolescent girls in a rural area of Odisha and its epidemiological correlates. Indian J Matern Child Health. 2013, 15:1-11. 